\section{PSS HEALTH: COMO CALCULAR TAMANHO DE AMOSTRA PARA ESTIMAR MÉDIA, PROPORÇÃO E CORRELAÇÃO}

\author{
PSS HEALTH: HOW TO CALCULATE A SAMPLE SIZE TO \\ ESTIMATE MEANS, PROPORTIONS, AND CORRELATIONS
}

\author{
Stela Maris de Jezus Castro ${ }^{1,2}$, Aline Castello Branco Mancuso', Suzi \\ Alves Camey ${ }^{1,2}$, Vanessa Bielefeldt Leotti ${ }^{1,2}$, Vânia Naomi Hirakata ${ }^{1}$, \\ Rogério Boff Borges ${ }^{1}$
}

\begin{abstract}
RESUMO
A ferramenta PSS Health (Power and Sample Size for Health Researchers) foi desenvolvida com o propósito de facilitar o cálculo do tamanho amostral e do poder de testes de hipóteses para diferentes objetivos de estudo, usando interface amigável e terminologia comum à área da saúde. Este é o primeiro de uma série de artigos que pretendem orientar o usuário na utilização da ferramenta PSS Health para o planejamento de uma pesquisa. Neste artigo, se ensina como utilizar o PSS Health quando o objetivo principal do estudo é estimar uma média, estimar uma proporção (prevalência ou incidência) ou estimar uma correlação. São disponibilizados vídeos demonstrando o uso da ferramenta em cada um dos contextos citados.
\end{abstract}

Palavras-chave: Tamanho de amostra; Estimação de parâmetros; R; Shiny

\begin{abstract}
The PSS Health (Power and Sample Size for Health Researchers) tool was developed with the purpose of facilitating the calculation of sample size and power of hypothesis tests for different study objectives, based on a user-friendly interface and common health care terminology. This is the first in a series of articles intending to guide the user in how to use the PSS Health tool for planning a research project. This article teaches how to use PSS Health when the main objective of the study is to estimate means, proportions (prevalence or incidence), or correlations. Videos showing how to use the tool in each of the mentioned contexts are available.
\end{abstract}

Keywords: Sample size, Parameter estimation, $R$, Shiny

\section{INTRODUÇÃO}

A ferramenta PSS Health (Power and Sample Size for Health Researchers), para cálculo do tamanho amostral e do poder de testes de hipóteses, foi apresentada à comunidade no artigo intitulado "Power and Sample Size for Health Researchers: uma ferramenta para cálculo de tamanho amostral e poder do teste voltado a pesquisadores da área da saúde", de Borges et al. ${ }^{1}$, e pode ser utilizada diretamente pelo computador através do software livre $\mathrm{R}^{2}$, com o pacote PSS.Health ${ }^{3}$, ou on-line no site da Unidade de Bioestatística (https://sites.google.com/hcpa.edu.br/bioestatistica) ou diretamente no endereço https://hcpa-unidade-bioestatistica.shinyapps.io/PSS_Health.

A criação desta ferramenta tem como principal motivação facilitar o cálculo de tamanho de amostra e poder de diversos projetos de pesquisa, através do uso de diferentes técnicas estatísticas. Na área da saúde, a maior parte dos estudos utiliza uma amostra representativa da população de interesse e os resultados encontrados na amostra são inferidos para a população. Esta etapa do estudo pode ser chamada de inferência estatística, a qual pode ser dividida
Clin Biomed Res. 2021;41(3):267-274 1 Unidade de Bioestatística, Grupo de Pesquisa e Pós-graduação (GPPG), Hospital de Clínicas de Porto Alegre (HCPA). Porto Alegre, RS, Brasil.

2 Departamento de Estatística, Instituto de Matemática e Estatística (IME), Universidade Federal do Rio Grande do Sul (UFRGS). Porto Alegre, RS, Brasil.

Autor correspondente: Stela Maris de Jezus Castro bioestatistica@hcpa.edu.br Unidade de Bioestatística, Hospital de Clínicas de Porto Alegre (HCPA) Rua Ramiro Barcelos, 2350 90035-007, Porto Alegre, RS, Brasil. 
em duas grandes áreas: estimação de parâmetros e testes de hipóteses sobre estes parâmetros.

Este artigo pretende orientar o usuário na utilização da ferramenta PSS Health para o planejamento de uma pesquisa quando o objetivo principal do estudo é estimar parâmetros estatísticos. Os parâmetros elencados aqui são: a média, a proporção (que também pode ser chamada de risco, prevalência ou incidência) e o coeficiente de correlação. Diferenças básicas no cálculo de tamanho de amostra entre estimação e testes de hipóteses podem ser vistas na resposta para a pergunta "Tamanho de amostra para teste de hipóteses e/ou para intervalo de confiança?" em Borges et al. ${ }^{4}$.

\section{ESTIMAÇÃO DE PARÂMETROS}

Em estatística, um parâmetro é a medida usada para descrever uma característica ou quantidade numérica populacional para uma variável de interesse, isto é, só poderia ser obtida se fosse avaliada em todos os elementos da população em estudo (o que caracteriza um censo). Se a variável de interesse for do tipo quantitativa, o parâmetro poderia ser a média da mesma (por exemplo, a média da idade em que ocorreu o diagnóstico de uma determinada doença) ou, se a variável for do tipo categórica, o parâmetro poderia ser a proporção de ocorrência da categoria de interesse da mesma (por exemplo, prevalência de fumantes, cujas categorias são "FUMANTE" e "NÃO FUMANTE"). Já o estimador do parâmetro populacional, também denominado estatística, é a descrição da mesma característica, porém determinada em uma amostra da população de interesse ${ }^{5}$. Por exemplo, a estimativa da média de idade de uma população é a média da idade observada em uma amostra.

A estimação de parâmetros pode ser realizada em duas formas: a estimação pontual e a estimação por intervalo de confiança. A estimativa por intervalo é útil em função da necessidade de se obter uma precisão da estimativa pontual para o parâmetro de interesse. Quando utilizada apenas a estimativa pontual, não há informação alguma sobre o quão próxima (ou distante) esta estimativa pode estar do verdadeiro valor do parâmetro estudado.

Assim, para o cálculo de tamanho de amostra em estudos onde o objetivo é a estimação de parâmetros, em geral o pesquisador precisará definir: o nível de confiança desejado para o intervalo de confiança (frequentemente 95\%); a margem de erro desejada, frequentemente informada como amplitude do intervalo de confiança ou semiamplitude (metade da largura total do intervalo de confiança); e uma medida prévia da variável de interesse. Uma medida prévia da variável de interesse como, por exemplo, a estimativa da variabilidade ou da proporção desejada, pode ser obtida em estudos anteriores onde esta variável foi mensurada (de preferência na mesma população alvo) ou de amostras piloto ${ }^{6}$.

\section{USO DO PSS HEALTH PARA CALCULAR O TAMANHO DE AMOSTRA}

\section{Tamanho de amostra para estimar uma média}

Suponha um estudo no qual o objetivo seja conhecer a pressão arterial sistólica média de crianças com excesso de peso no primeiro ano de vida em acompanhamento em um determinado hospital. Para tanto, será selecionada uma amostra aleatória simples de crianças em atendimento neste hospital durante o período de 2 anos e o pesquisador precisa saber quantas devem ser selecionadas para fazer parte da amostra, ou seja, o pesquisador precisa saber o tamanho da amostra (exemplo adaptado de Vieira et al. ${ }^{7}$ ).

As informações requeridas para o cálculo do tamanho de amostra para este estudo são:

1. Nível de confiança desejado para o intervalo de confiança;

2. Margem de erro desejada para o intervalo de confiança (semiamplitude absoluta do intervalo de confiança);

3. Desvio padrão esperado para a variável pressão sistólica (desfecho) na população de crianças com excesso de peso em seu primeiro ano de vida.

Para calcular o tamanho da amostra para estimar uma média no site do PSS Health, deve-se clicar na aba "MÉDIAS" e em seguida na opção "UMA AMOSTRA" (Figura 1).

Médias Proporçōes Qui-quadrado

Uma amostra

Dois grupos independentes

Dois grupos independentes (Inf/ Equi/ Sup)

Dois grupos dependentes

Medidas repetidas

ANOVA de uma via

ANOVA de duas vias

Figura 1: Aba do PSS Health para cálculo de tamanho de amostra e poder para o parâmetro média. 
Ao selecionar "UMAAMOSTRA" aparecerão três abas com as opções de cálculo de tamanho de amostra para estimar uma média, para testar uma média e uma aba com a opção para cálculo de poder. Clicando na aba "ESTIMAR" as caixas com as informações requeridas para o cálculo de tamanho de amostra para estimar uma média irão aparecer (Figura 2). Observe que, além das informações elencadas anteriormente (itens 1, 2 e 3), é necessário informar a opção do processo de amostragem aleatória. Dentre os demais campos apresentados, também é possível descrever o nome do desfecho e a unidade de medida deste desfecho para edição do parágrafo a ser sugerido pelo programa (Figura 3). Por fim, há uma caixa para incluir no cálculo um percentual de perdas ou recusas, caso seja de interesse. Em caso de dúvida sobre as informações requeridas em cada campo, pode-se clicar na interrogação apresentada ao lado do mesmo e obter uma ajuda quanto à sua definição e como preencher. A Figura 2 mostra as informações para o exemplo: o processo de amostragem aleatória simples, desfecho definido como Pressão Arterial Sistólica (medido em $\mathrm{mmHg}$ ), precisão do intervalo de confiança definida como uma margem de erro igual a $2 \mathrm{mmHg}$, desvio padrão do desfecho na população alvo do estudo igual a $9.58 \mathrm{mmHg}$ (obtido em Vieira et al. ${ }^{7}$ ), nível de confiança desejado para o intervalo igual a 95\%, e uma margem de segurança para possíveis perdas ou recusas entre as unidades amostrais igual a $10 \%$.

Preenchidos os campos solicitados, ao lado direito da janela, em destaque na parte superior, será apresentado o tamanho de amostra calculado (Figura 3), conforme as definições estabelecidas na Figura 2. Para o exemplo, são necessárias 91 crianças com excesso de peso no primeiro ano de vida para estimar a pressão sistólica média com $95 \%$ de confiança e margem de erro de $2 \mathrm{mmHg}$. Logo abaixo é apresentada uma sugestão de texto para a descrição do cálculo realizado, sendo ainda necessário substituir o termo "Fulano (1900)" pela referência da informação sobre o desvio padrão utilizado no cálculo do tamanho da amostra. Neste exemplo, esta informação foi retirada de Vieira et al. ${ }^{7}$.
A última frase do texto sugerido se refere ao tamanho de amostra que considera os $10 \%$ de perdas ou recusas que possam ocorrer durante a coleta (neste exemplo, o tamanho da amostra aumenta de 91 para 102 crianças). Ainda na Figura 3, há uma sugestão de como citar a ferramenta PSS Health e a descrição do pacote e comando do software $R$ utilizado no cálculo.

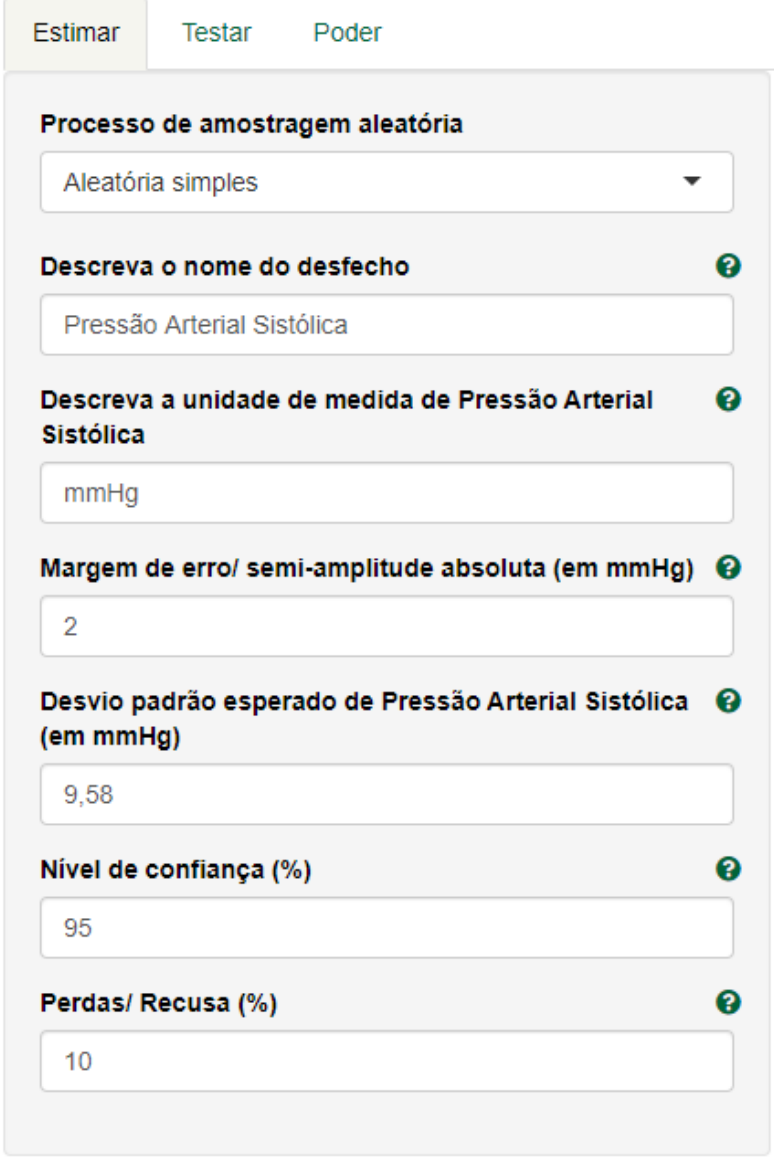

Figura 2: Aba do PSS Health contendo os campos já preenchidos para o cálculo de tamanho de amostra para estimar a pressão arterial sistólica média de acordo com o exemplo. 
Tamanho amostral calculado: 91

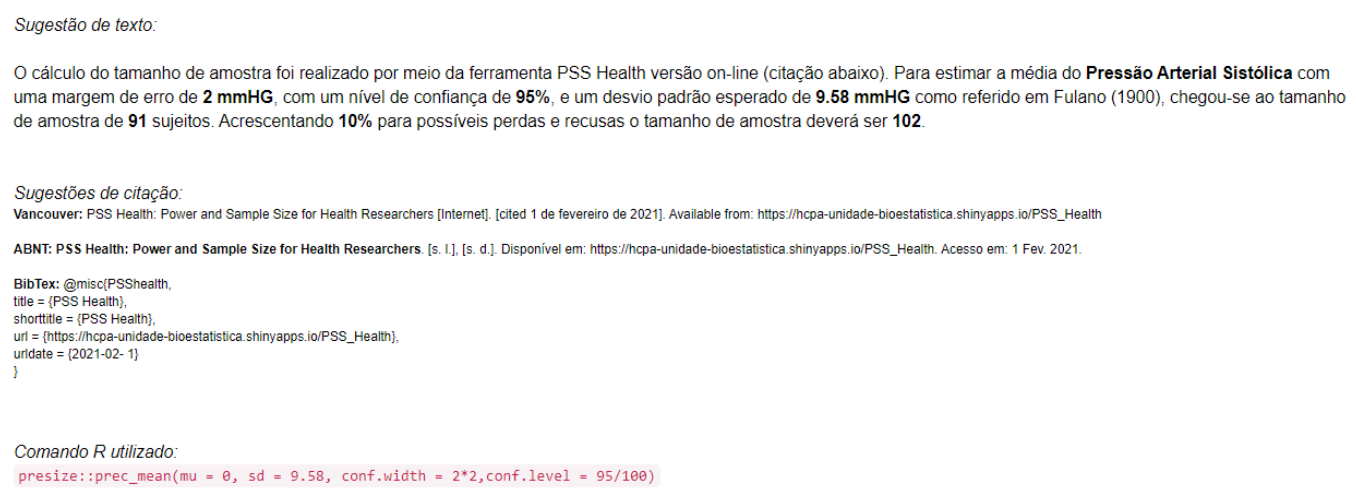

presize: : prec_mean $(m u=\theta$, sd $=9.58$, conf.width $=2 * 2$, conf.level $=95 / 100)$

* Sempre procure um profissional de estatistica para orientaçöes no planejamento do estudo.

Figura 3: Resultado do cálculo de tamanho de amostra obtido a partir dos campos preenchidos na Figura 2.

Outra utilidade oferecida pelo PSS Health na opção de cálculo amostral é a possibilidade de construir cenários de tamanhos de amostra. Os cenários podem ser visualizados através de um gráfico (Figura 4) e através de uma tabela (Figura 5), sendo permitido o download dos dois. No exemplo, pode-se variar a precisão (margem de erro) para diferentes valores de desvio padrão. É importante lembrar que a margem de erro é diretamente proporcional ao erro-padrão que, por sua vez, é diretamente proporcional ao desvio-padrão da variável. Ou seja, quanto maior o desvio-padrão, maior será a margem de erro. Como pode ser visualizado nas Figuras 4 e 5, ao aumentar a margem de erro reduzimos o tamanho da amostra.
Ocasionalmente, o desvio padrão da variável de interesse não pode ser obtido na literatura (ou de uma amostra piloto), mas se pode encontrar outras estatísticas, tais como erro padrão, intervalo de confiança, valor de t ou valor de p. Nesta situação, o PSS Health, na aba 'Outras ferramentas', permite que se faça a conversão destas medidas em uma medida de desvio padrão. Caso as medidas descritivas disponíveis na literatura sejam o mínimo, o primeiro quartil, a mediana, o terceiro quartil, o máximo e o tamanho da amostra (n), formas de obtenção de estimativas do desvio padrão a partir de um subconjunto destas medidas (por exemplo, mínimo, mediana, máximo e n) podem ser encontradas em Wan et al. ${ }^{8}$.

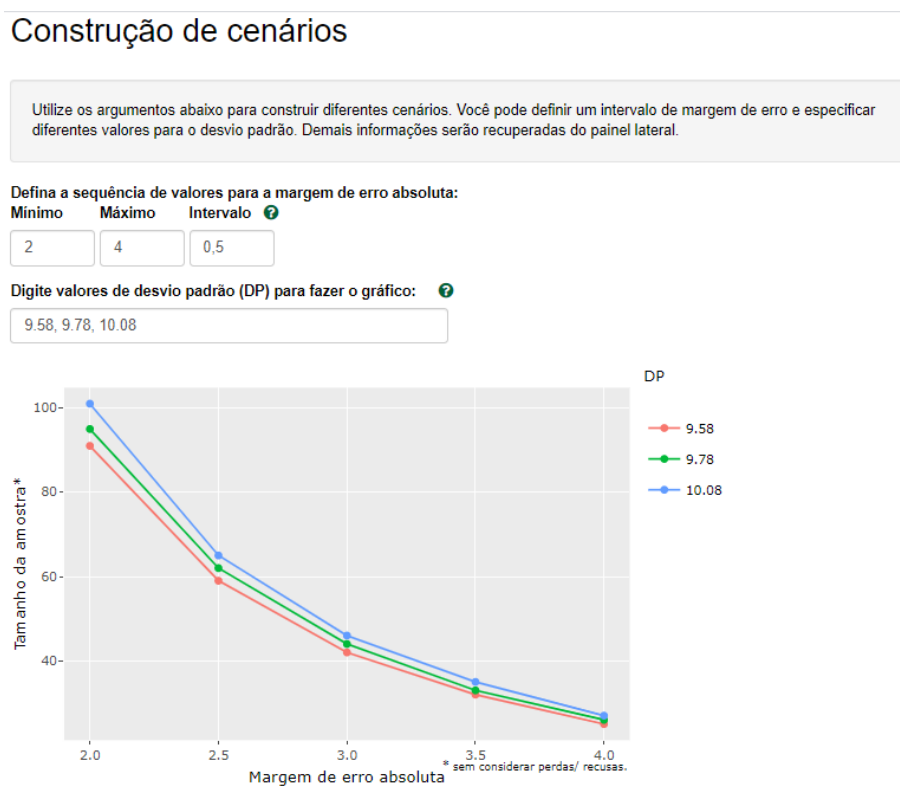

Figura 4: Representação gráfica da construção de cenários. 


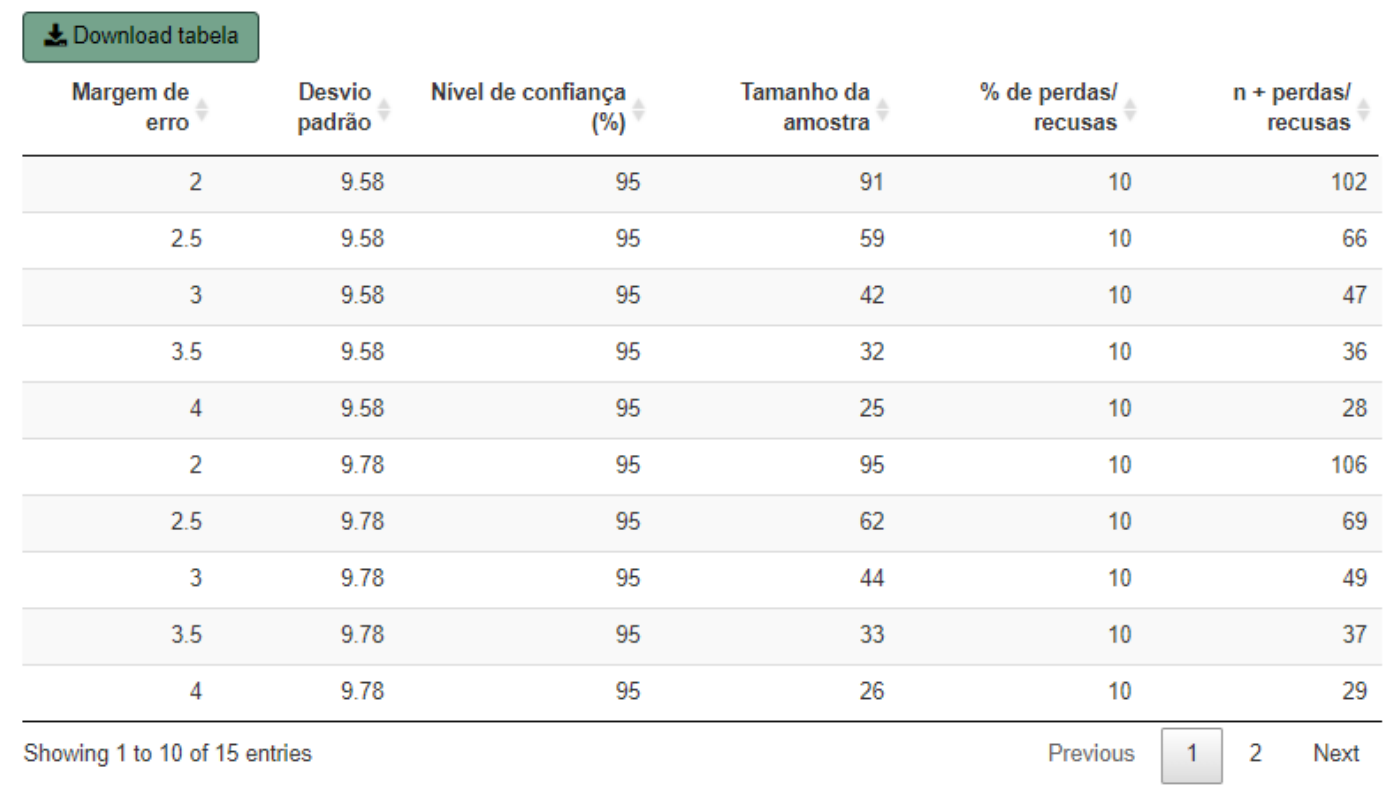

Figura 5: Tabela com os resultados da construção de cenários.

O exemplo de como utilizar o PSS Health para calcular o tamanho de amostra para estimar uma média também pode ser visualizado no vídeo "PSS para Estimar uma Média"9.

Observa-se, ainda, que no exemplo em questão foi utilizada a opção de amostragem aleatória simples. No entanto, o PSS Health também possibilita o cálculo do tamanho de amostra para estimar uma média para os delineamentos amostrais conhecidos como "Conglomerados em um único estágio" e "Estratificada proporcional ao tamanho". Em ambos delineamentos são requeridas outras informações para o cálculo do tamanho de amostra, além daquelas necessárias no caso da amostragem aleatória simples (informações sobre estes delineamentos amostrais podem ser obtidas em Bolfarine e Bussab ${ }^{10}$ ).

Vale lembrar que selecionar uma amostra aleatória simples implica em basicamente sortear os participantes a partir de uma listagem. Entretanto há situações nas quais o pesquisador não possui essa listagem e opta pela chamada amostra por conveniência onde, por exemplo, o pesquisador seleciona para sua amostra uma sequência de pacientes que chegam em um ambulatório buscando atendimento. A amostra por conveniência é um método não probabilístico e, portanto, nenhum dos conceitos aqui descritos podem ser aplicados. Isso significa que não faz sentido falar em margem de erro ou confiança de um intervalo para esse tipo de amostra, pois são conceitos baseados na hipótese de que todos os pacientes tiveram a mesma probabilidade de serem escolhidos para a amostra. Uma maneira de contornar a falta de uma listagem, mas chegar próximo de garantir que todos os pacientes tenham a mesma probabilidade de serem escolhidos é, por exemplo, fazer uma grade com os horários de atendimento e sortear em quais os horários ocorrerão as entrevistas. Outra possibilidade é, para cada dia da semana, sortear o horário do primeiro entrevistado e a partir deste fazer saltos de tempo para as próximas entrevistas.

\section{Tamanho de amostra para estimar uma proporção}

Suponha um estudo no qual o objetivo seja conhecer a prevalência (proporção) de perda da acuidade auditiva em idosos com risco de vulnerabilidade clínico-funcional, mensurada através do teste do Sussurro. Para tanto, será selecionada uma amostra aleatória simples destes idosos e o pesquisador precisa saber quantos devem ser selecionados para fazer parte da amostra (exemplo adaptado de Freitas e Soares ${ }^{11}$ ).

As informações requeridas para o cálculo do tamanho de amostra para este estudo são:

1. Nível de confiança desejado para o intervalo;

2. A amplitude do intervalo de confiança em pontos percentuais, ou seja, a margem de erro desejada para o intervalo de confiança multiplicada por dois;

3. A prevalência (proporção) esperada de ocorrência da perda de acuidade auditiva em idosos com risco de vulnerabilidade clínico-funcional, mensurada através do teste do Sussurro. 


\section{Proporções - Qui-quadrado Correlação}

Uma amostra

Dois grupos independentes

Dois grupos independentes (Inf/ Equi/ Sup)

Dois grupos dependentes

Figura 6: Aba do PSS Health para cálculo de tamanho de amostra e poder para o parâmetro proporção.

Para calcular o tamanho de amostra para estimar uma prevalência no PSS Health, deve-se clicar na aba "PROPORÇÕES" e em seguida na opção "UMA AMOSTRA" (Figura 6). Aparecerão, então, as abas com as opções de cálculo de tamanho de amostra para estimar ou testar uma proporção e uma aba com a opção para cálculo de poder. Clicando na aba "ESTIMAR", as caixas com as informações requeridas para o cálculo de tamanho de amostra para estimar uma proporção irão aparecer. As informações requeridas para o cálculo de tamanho de amostra para o exemplo são: nome do desfecho (Perda de Acuidade Auditiva), a amplitude do intervalo de confiança igual a $10 \%$, percentual esperado de perda de acuidade auditiva em idosos com risco de vulnerabilidade clínico-funcional igual a $40,5 \%$ (obtido em Freitas e Soares ${ }^{11}$ ), nível de confiança desejado para o intervalo igual a 95\%, e uma margem de segurança para possíveis perdas ou recusas entre as unidades amostrais igual a $10 \%$. Deve-se, ainda, selecionar o método para calcular o intervalo de confiança, o PSS Health oferece quatro diferentes métodos: Wilson, Agresti-Coull, exact e Wald $^{12}$. Detalhes sobre o uso destes métodos podem ser obtidos na documentação do pacote presize ${ }^{13}$ ).
No exemplo, manteremos o método de Wilson. O vídeo "PSS para Estimar uma Proporção"14 mostra como calcular o tamanho da amostra para este exemplo utilizando o PSS Health.

Em situações onde o percentual de ocorrência da categoria de interesse esperado não pode ser obtido na literatura, sugere-se utilizar o valor de $50 \%$, pois, para este percentual, a variabilidade é máxima para o estimador da proporção. Ou seja, garantese assim, o maior tamanho da amostra necessário para a estimativa (mantendo o nível de confiança e a amplitude do intervalo fixos).

\section{Tamanho de amostra para estimar um coefici- ente de correlação}

Suponha um estudo no qual o objetivo seja conhecer a correlação entre um indicador de adiposidade corporal (IMC) e o escore de consumo de padrão alimentar tradicional em crianças de 4 a 7 anos. Para tanto, será selecionada uma amostra aleatória simples destas crianças e o pesquisador precisa saber quantas devem ser selecionadas para fazer parte da amostra (exemplo adaptado de Vieira ${ }^{15}$ ).

As informações requeridas para o cálculo do tamanho de amostra para este estudo são:

1. Nível de confiança desejado para o intervalo;

2. Definição de qual o coeficiente de correlação desejado (Pearson, Spearman ou Kendall);

3. O coeficiente de correlação esperado (ou o mínimo considerado clinicamente relevante pelo pesquisador);

4. A amplitude do intervalo de confiança (margem de erro desejada para o intervalo de confiança multiplicada por dois).

\section{Coeficientes de correlação}

Muitas vezes o objetivo do estudo é analisar se duas variáveis variam conjuntamente. Nestes casos, a estatística de interesse é o coeficiente de correlação. Os coeficiente de correlação avaliam a direção e o grau de alinhamento entre duas variáveis. Assume valores que variam de -1 (correlação negativa perfeita) a 1 (correlação positiva perfeita). Vale ressaltar que, para realizar inferências para o coeficiente de correlação linear de Pearson, é necessário supor que os dados aderem à uma distribuição normal e possuem variância constante em torno da reta de tendência.

Figura 7: Aba do PSS Health para cálculo de tamanho de amostra para se estimar uma proporção. 
Para calcular o tamanho de amostra para estimar um coeficiente de correlação no PSS Health, deve-se clicar na aba "CORRELAÇÃO" (Figura 7), onde aparecerão as abas com as opções de cálculo de tamanho de amostra para estimar ou testar um coeficiente de correlação, e uma aba com a opção para cálculo de poder, assim como nos exemplos anteriores. Clicando na aba "ESTIMAR", as caixas com as informações requeridas para o cálculo de tamanho de amostra para estimar um coeficiente de correlação irão aparecer. Primeiramente, o pesquisador deve definir qual o coeficiente de correlação desejado, pois o PSS Health permite calcular o tamanho de amostra para o coeficiente de correlação de Pearson, de Spearman ou Kendall.

Para o exemplo se adotará o coeficiente de Spearman, o mesmo utilizado na referência, e as informações requeridas para o cálculo de tamanho de amostra são: nomes das duas variáveis para as quais se deseja estimar o coeficiente de correlação (escore de consumo alimentar tradicional e índice de massa corporal - IMC), coeficiente de correlação esperado entre as duas variáveis que é de 0,13 (obtido em Vieira ${ }^{15}$ ), amplitude do intervalo de confiança igual a 0,4 , nível de confiança desejado para o intervalo igual a 95\% e uma margem de segurança para possíveis perdas ou recusas entre as unidades amostrais igual a $10 \%$. O vídeo "PSS para Estimar uma Correlação" 16 mostra como calcular o tamanho da amostra para este exemplo utilizando o PSS Health.

Nas situações onde se deseja estimar o coeficiente de correlação entre diversas variáveis, por exemplo, entre escore de consumo alimentar tradicional e indicadores de adiposidade corporal (IMC), relação cintura-estatura, percentual de gordura corporal e percentual de gordura central, deve-se utilizar no cálculo do tamanho de amostra o menor coeficiente de correlação esperado entre as variáveis, pois quanto menor o coeficiente, maior será o tamanho da amostra. Deste modo, garante-se o maior tamanho da amostra necessário para estimar todos os coeficientes do estudo (mantendo o nível de confiança e a amplitude do intervalo fixos).

\section{REFERÊNCIAS}

1. Borges RB, Mancuso ACB, Camey SA, Leotti VB, Hirakata VN, Azambuja GS, et al. Power and Sample Size for Health Researchers: uma ferramenta para cálculo de tamanho amostral e poder do teste voltado a pesquisadores da área da saúde. Clin Biomed Res [Internet]. 2020 [citado 21 maio 2021];40(4):247253. Disponível em: https://seer.ufrgs. br/hcpa/article/view/109542

2. R Core Team. R: A Language and Environment for Statistical Computing [Internet]. Vienna: $R$ Foundation for Statistical Computing; 2020. Disponível em: https://www.Rproject.org/.

3. Borges RB, Azambuja GS, Mancuso ACB, Leotti VB, Hirakata VN, Camey SA, et al. PSS. Health: Power and Sample Size for Health Researchers via Shiny [Internet]. Porto Alegre:Unidade de Bioestatística do Hospital das Clínicas de Porto Alegre; 2020 [citado 5 fev. 2021]. Disponível em: https://CRAN.R-project.org/ package $=$ PSS. Health

4. Borges RB, Leotti VB, Mancuso ACB, Castro SMJ, Hirakata VN, Camey SA. Equívocos Estatísticos: Perguntas que você sempre quis fazer, mas nunca teve coragem. Clin Biomed
Res [Internet]. 15 jul. 2020 [citado 6 nov2020];40(1):63-70. Disponível em: https://seer.ufrgs.br/hcpa/article/ view/101299

5. Morettin LG. Estatística básica: probabilidade e inferência. São Paulo: Makron; 2010.

6. Hulley SB, Browner WS Cummings SR, Grady D, Newman TB. Delineando a pesquisa clínica: uma abordagem epidemiológica [Internet]. Porto Alegre: Artmed; 2008. Disponível em: http://books.google.com.br/ books?id=bshuPgAACAAJ

7. Vieira SA, Fonseca PCA, Andreoli CS, Pereira PF, Hermsdorff $\mathrm{HHM}$, Ribeiro $A Q$, et al. A pressão arterial está associada a indicadores de adiposidade corporal em crianças de quatro a sete anos. Rev Port Cardiol. 2018;37(5):425-32.

8. Wan X, Wang W, Liu J, Tong T. Estimating the sample mean and standard deviation from the sample size, median, range and/or interquartile range. BMC Med Res Methodol. 19 dez 2014;14(1):135.

9. PSS para Estimar uma Média.mp4 [Internet]. Porto Alegre: Hospital de Clínicas de Porto Alegre, Unidade de Bioestatística; [2021?] [citado 23 ju
2021]. Vídeo: 5:30 min. Disponível em: https://drive.google.com/ file/d/1MJ71kOWOv9U1MLcvzMLSDIEZzzvp3WB/view?usp= sharing\&usp=embed_facebook

10. Bolfarine $\mathrm{H}$, Bussab WO. Elementos de Amostragem. São Paulo: Blucher; 2005.

11. Freitas FFQ, Soares SM. Índice de vulnerabilidade clínico-funcional e as dimensões da funcionalidade em idosos. Rev Rene. 20 maio 2019; 20:e39746.

12. Brown LD, Cai TT, DasGupta A. Interval estimation for a binomial proportion. Statist Sci. 2001;16(2):101-33.

13. Lenz A, Haynes AG, Limacher A, Stalder O. Presize: Precision Based Sample Size Calculation [Internet]. [local desconhecido: editora desconhecida]; 2020 [citado 9 mar. 2021]. Disponível em: https://CRAN.R-project.org/ package $=$ presize

14. PSS para Estimar uma Proporção. mp4 [Internet]. Porto Alegre: Hospital de Clínicas de Porto Alegre, Unidade de Bioestatística; [2021?] [citado 23 jul 2021]. Vídeo: 2:58 min. Disponível em: https://drive.google.com/file/d/19 
Sp4HjcwdwrDwR2Qie3XCk8zuZz20JNs/ view?usp=sharing\&usp= embed_facebook

15. Vieira SA. Padrão alimentar, adiposidade corporal e fatores de risco cardiometabólico em crianças de 4 a 7 anos de idade [tese].
Viçosa: Universidade Federal de Viçosa; 2017 [citado 11 mar 2021]. Disponível em: https://locus.ufv.br// handle/123456789/10455

16. 16. PSS para Estimar Correlação. mp4 [Internet]. Porto Alegre: Hospital de Clínicas de Porto Alegre, Unidade de Bioestatística; [2021?] [citado 23 jul 2021]. Vídeo: 3:55 min. Disponível em: https://drive.google. com/file/d/1NrLvKZI6WyKwqcy0mdPMOoq7sqZDNWx/ view? $\mathrm{usp}=$ sharing\&us $p=$ embed facebook 